Supplement of Atmos. Chem. Phys., 17, 6073-6089, 2017

http://www.atmos-chem-phys.net/17/6073/2017/

doi:10.5194/acp-17-6073-2017-supplement

(C) Author(s) 2017. CC Attribution 3.0 License.

(c) (i)

Supplement of

\title{
Ozone and haze pollution weakens net primary productivity in China
}

Xu Yue et al.

Correspondence to: Xu Yue (xuyueseas@gmail.com)

The copyright of individual parts of the supplement might differ from the CC-BY 3.0 licence. 
Table S1. Summary of measurements for ozone damage to photosynthesis a

\begin{tabular}{|c|c|c|c|c|c|c|}
\hline \multirow{2}{*}{ PFT } & \multirow{2}{*}{ Species } & \multirow{2}{*}{$\begin{array}{c}{\left[\mathrm{O}_{3}\right]} \\
(\mathrm{ppbv})\end{array}$} & \multicolumn{2}{|c|}{ Damage (\%) } & \multirow{2}{*}{$\operatorname{Sym}^{c}$} & \multirow{2}{*}{ Reference } \\
\hline & & & Mean & Range & & \\
\hline \multirow{4}{*}{ All } & \multirow{4}{*}{ Meta-analysis } & 37.5 & -8.5 & {$[-17.5,0.5]$} & \multirow{4}{*}{$\mathrm{L}$} & \multirow{4}{*}{$\begin{array}{l}\text { Lombardozz } \\
\text { et al. (2013) }\end{array}$} \\
\hline & & 62.5 & -24.5 & {$[-29,-20]$} & & \\
\hline & & 87.5 & -15 & {$[-22,-8]$} & & \\
\hline & & 112.5 & -19.5 & {$[-33,-6]$} & & \\
\hline \multirow{4}{*}{ ENF } & \multirow{2}{*}{ Picea / Pinus } & 45 & -2 & {$[-6,3]$} & \multirow{2}{*}{ W } & \multirow{2}{*}{$\begin{array}{l}\text { Wittig et al. } \\
\text { (2007) }\end{array}$} \\
\hline & & 92 & -17 & {$[-21,-12]$} & & \\
\hline & $\begin{array}{l}\text { Cinnamomum camphora } \\
\text { /Cyclobalanopsis glauca / } \\
\text { Neolitsea sericea/ } \\
\text { Schima superba }\end{array}$ & 150 & -26 & {$[-32,-16]$} & $\mathbf{Z}$ & $\begin{array}{l}\text { Zhang et al. } \\
\text { (2012b) }\end{array}$ \\
\hline & $\begin{array}{c}\text { Pinus tabulaeformis / } \\
\text { Pinus armandii }\end{array}$ & 85 & -33.8 & {$[-36.7,-31.0]$} & $\mathbf{X}$ & $\begin{array}{l}\text { Xu et al. } \\
\text { (2015) }\end{array}$ \\
\hline \multirow{6}{*}{ DBF } & Betula / Fagus / & 48 & -14 & {$[-16,-12]$} & \multirow{2}{*}{ W } & \multirow{2}{*}{$\begin{array}{l}\text { Wittig et al. } \\
\text { (2007) }\end{array}$} \\
\hline & $\begin{array}{c}\text { Lirlodendron / Populus } \\
\text { Prunus / Quercus }\end{array}$ & 82 & -20 & {$[-23,-16]$} & & \\
\hline & Ginkgo biloba & 80 & -21 & {$[-53,-5]$} & $\mathbf{H}$ & $\begin{array}{l}\text { He et al. } \\
(2007)\end{array}$ \\
\hline & Cinnamomum camphora & 95 & -28.0 & {$[-31.7,-20.4]$} & $\mathbf{F}$ & $\begin{array}{c}\text { Feng et al. } \\
\text { (2011a) }\end{array}$ \\
\hline & $\begin{array}{l}\text { Liriodendron chinense / } \\
\text { Liquidambar formosana }\end{array}$ & 150 & -39 & {$[-42,-36]$} & $\mathbf{Z}$ & $\begin{array}{c}\text { Zhang et al. } \\
(2012 b)\end{array}$ \\
\hline & $\begin{array}{c}\text { Ginkgo biloba / Quercus } \\
\text { mongolica }\end{array}$ & 85 & -34 & {$[-51.5,-17]$} & $\mathbf{X}$ & $\begin{array}{l}\text { Xu et al. } \\
\text { (2015) }\end{array}$ \\
\hline \multirow{5}{*}{ SHRUB } & Calluna vulgaris & 70 & -7.1 & {$[-16.4,-1.7]$} & Fo & $\begin{array}{c}\text { Foot et al. } \\
(1996)\end{array}$ \\
\hline & $\begin{array}{l}\text { Phillyrea latifolia L./ } \\
\text { Arbutus unedo L./ } \\
\text { Laurus nobilis L. }\end{array}$ & $68^{\mathrm{b}}$ & -6.4 & {$[-8.8,-1.9]$} & $\mathrm{N}$ & $\begin{array}{l}\text { Nali et al. } \\
\text { (2004) }\end{array}$ \\
\hline & $\begin{array}{c}\text { Pistacia terebinthus / } P . \\
\text { lentiscus / Viburnum } \\
\text { lantana /V. tinus }\end{array}$ & 76 & -16.3 & {$[-26.5,-9.5]$} & $\mathrm{C}$ & $\begin{array}{l}\text { Calatayud et } \\
\text { al. }(2010)\end{array}$ \\
\hline & $\begin{array}{l}\text { Ilex integra } / \\
\text { Photinia } \times \text { fraseri }\end{array}$ & 150 & -4.5 & {$[-6,-3]$} & $\mathbf{Z}$ & $\begin{array}{l}\text { Zhang et al. } \\
\text { (2012b) }\end{array}$ \\
\hline & $\begin{array}{l}\text { Euonymus bungeanus } \\
\text { Maxim. / Photinia } \times \\
\text { fraseri / Chionanthus } \\
\text { retusus Lindl. \& Paxt. / } \\
\text { Cornus alba L. }\end{array}$ & 70 & -43.1 & {$[-49.6,-36.5]$} & $\mathbf{Z}$ & $\begin{array}{l}\text { Zhang et al. } \\
\text { (2012a) }\end{array}$ \\
\hline
\end{tabular}




\begin{tabular}{|c|c|c|c|c|c|c|}
\hline \multirow{10}{*}{ HERB_C3 } & \multirow{2}{*}{ Soybean } & 38 & 0.2 & {$[-4.5,6.5]$} & \multirow{2}{*}{ M } & \multirow{2}{*}{$\begin{array}{l}\text { Mulchi et al. } \\
\quad(1992)\end{array}$} \\
\hline & & 84 & -11.3 & {$[-19.4,-6.8]$} & & \\
\hline & \multirow{5}{*}{ Wheat } & 73 & -20 & {$[-24,-20]$} & $\mathrm{F}$ & $\begin{array}{c}\text { Feng et al. } \\
(2008)\end{array}$ \\
\hline & & 82 & -24 & {$[-35,-12]$} & B & $\begin{array}{c}\text { Biswas et al. } \\
\text { (2008) }\end{array}$ \\
\hline & & 55 & -12.9 & {$[-18.4,-7.3]$} & $\mathbf{F}$ & $\begin{array}{c}\text { Feng et al. } \\
\text { (2011b) }\end{array}$ \\
\hline & & 100 & -18.3 & {$[-38.2,-8.2]$} & \multirow{2}{*}{$\mathbf{Z e}$} & \multirow{2}{*}{$\begin{array}{l}\text { Zheng et al. } \\
\quad \text { (2010) }\end{array}$} \\
\hline & & 150 & -25.9 & {$[-42.5,-5.8]$} & & \\
\hline & \multirow{2}{*}{ Rice } & 59 & -28 & {$[-34,-22]$} & A & $\begin{array}{l}\text { Ainsworth } \\
(2008)\end{array}$ \\
\hline & & 54 & -15.6 & {$[-22.7,-8.4]$} & $\mathbf{P}$ & $\begin{array}{c}\text { Pang et al. } \\
\text { (2009) }\end{array}$ \\
\hline & Snap bean & 71 & -22.0 & {$[-38.8,-4.5]$} & $\mathbf{Y}$ & $\begin{array}{c}\text { Yuan et al. } \\
\text { (2015) }\end{array}$ \\
\hline \multirow{4}{*}{ HERB_C4 } & Spartina alterniflora & 80 & -23.7 & {$[-34.7,-8.3]$} & $\mathrm{T}$ & $\begin{array}{l}\text { Taylor et al. } \\
\text { (2002) }\end{array}$ \\
\hline & \multirow{2}{*}{ Sugarcane clones } & 76 & -25.9 & {$[-38.1,-9.3]$} & \multirow{2}{*}{$\mathrm{G}$} & \multirow{2}{*}{$\begin{array}{l}\text { Grantz et al. } \\
\quad(2012)\end{array}$} \\
\hline & & 147 & -50 & {$[-73.7,-17.9]$} & & \\
\hline & Maize & 80 & -23.5 & {$[-33.7,-16.8]$} & $\mathbf{F u}$ & $\begin{array}{c}\text { Fu et al. } \\
(2008)\end{array}$ \\
\hline
\end{tabular}

${ }^{a}$ Bold references are experiments performed for species in China. $\mathrm{All}_{3}$ concentrations are averaged for an 8-hour exposure period per day. Most of these statistics are derived based on multiple literature sources, species, or genotypes.

${ }^{b}$ The average $\left[\mathrm{O}_{3}\right]$ is for 8 -hour average converted from the 5-hour exposure at $110 \mathrm{ppbv}$.

${ }^{c}$ The abbreviation of reference shown as symbol on Figure 5. 
Table S2. Summary of 15 simulations with offline vegetation model YIBs

\begin{tabular}{cccc}
\hline ID & Simulations $^{\text {a }}$ & {$\left[\mathrm{O}_{3}\right](\mathrm{ppbv})$} & $\mathrm{O}_{3}$ damage $^{\mathrm{b}}$ \\
\hline 1 & YIBS_O000 & 0 & Null \\
2 & YIBS_O020L & 20 & Low \\
3 & YIBS_O020H & 20 & High \\
4 & YIBS_O040L & 40 & Low \\
5 & YIBS_O040H & 40 & High \\
6 & YIBS_O060L & 60 & Low \\
7 & YIBS_O060H & 60 & High \\
8 & YIBS_O080L & 80 & Low \\
9 & YIBS_O080H & 80 & High \\
10 & YIBS_O100L & 100 & Low \\
11 & YIBS_O100H & 100 & High \\
12 & YIBS_O120L & 120 & Low \\
13 & YIBS_O120H & 120 & High \\
14 & YIBS_O140L & 140 & Low \\
15 & YIBS_O140H & 140 & High \\
\hline
\end{tabular}

${ }^{a}$ Each simulation is performed for 1995-2011. The last ten years are used to calculate the ozone-induced damages to gross primary productivity (GPP).

${ }^{\mathrm{b}}$ Ozone damage applied in the simulation can be low or high for the same level of $\left[\mathrm{O}_{3}\right]$, depending on the selection of damaging coefficients (Sitch et al., 2007). 
Table S3. Summary of 30 simulations using offline vegetation model YIBs driven with simulated meteorology from the climate model ModelE2-YIBs

\begin{tabular}{|c|c|c|c|c|}
\hline Simulations & Base forcing & Temperature & PAR & Soil moisture \\
\hline YG10_NAT & G10NATNO3 & & & \\
\hline YG10_ALL & G10NATNO3 & G10ALLNO3 & G10ALLNO3 & G10ALLNO3 \\
\hline YG10_TAS & G10NATNO3 & G10ALLNO3 & & \\
\hline YG10_PAR & G10NATNO3 & & G10ALLNO3 & \\
\hline YG10_SLM & G10NATNO3 & & & G10ALLNO3 \\
\hline YG30_NAT & G30NATNO3 & & & \\
\hline YG30_ALL & G30NATNO3 & G30ALLNO3 & G30ALLNO3 & G30ALLNO3 \\
\hline YG30_TAS & G30NATNO3 & G30ALLNO3 & & \\
\hline YG30_PAR & G30NATNO3 & & G30ALLNO3 & \\
\hline YG30_SLM & G30NATNO3 & & & G30ALLNO3 \\
\hline YM30_NAT & M30NATNO3 & & & \\
\hline YM30_ALL & M30NATNO3 & M30ALLNO3 & M30ALLNO3 & M30ALLNO3 \\
\hline YM30_TAS & M30NATNO3 & M30ALLNO3 & & \\
\hline YM30_PAR & M30NATNO3 & & M30ALLNO3 & \\
\hline YM30_SLM & M30NATNO3 & & & M30ALLNO3 \\
\hline YG10AIE_NAT & G10NATNO3_AIE & & & \\
\hline YG10AIE_ALL & G10NATNO3_AIE & G10ALLNO3_AIE & G10ALLNO3_AIE & G10ALLNO3_AIE \\
\hline YG10AIE_TAS & G10NATNO3_AIE & G10ALLNO3_AIE & & \\
\hline YG10AIE_PAR & G10NATNO3_AIE & & G10ALLNO3_AIE & \\
\hline YG10AIE_SLM & G10NATNO3_AIE & & & G10ALLNO3_AIE \\
\hline YG30AIE_NAT & G30NATNO3_AIE & & & \\
\hline YG30AIE_ALL & G30NATNO3_AIE & G30ALLNO3_AIE & G30ALLNO3_AIE & G30ALLNO3_AIE \\
\hline YG30AIE_TAS & G30NATNO3_AIE & G30ALLNO3_AIE & & \\
\hline YG30AIE_PAR & G30NATNO3_AIE & & G30ALLNO3_AIE & \\
\hline YG30AIE_SLM & G30NATNO3_AIE & & & G30ALLNO3_AIE \\
\hline YM30AIE_NAT & M30NATNO3_AIE & & & \\
\hline YM30AIE_ALL & M30NATNO3_AIE & M30ALLNO3_AIE & M30ALLNO3_AIE & M30ALLNO3_AIE \\
\hline YM30AIE_TAS & M30NATNO3_AIE & M30ALLNO3_AIE & & \\
\hline YM30AIE_PAR & M30NATNO3_AIE & & M30ALLNO3_AIE & \\
\hline YM30AIE_SLM & M30NATNO3_AIE & & & M30ALLNO3_AIE \\
\hline
\end{tabular}


Table S4. Comparison of simulated $\mathrm{O}_{3}$ concentrations with measurements at Chinese non-urban sites

\begin{tabular}{|c|c|c|c|c|c|}
\hline \multirow{2}{*}{ Locations } & \multirow{2}{*}{ Time period } & \multirow{2}{*}{ Season } & \multicolumn{2}{|c|}{$\left[\mathrm{O}_{3}\right]$ (ppbv) } & \multirow{2}{*}{ Reference } \\
\hline & & & Obs. & Model & \\
\hline \multirow{3}{*}{$\begin{array}{c}\text { Longfengshan } \\
\left(44.7^{\circ} \mathrm{N}, 127.6^{\circ} \mathrm{E}\right)\end{array}$} & \multirow{3}{*}{2006} & Spring & 43 & 47.3 & \multirow{3}{*}{ An et al. (2013) } \\
\hline & & Summer & 33 & 42.4 & \\
\hline & & Fall & 29 & 38.0 & \\
\hline \multirow{3}{*}{$\begin{array}{c}\text { Shangdianzi } \\
\left(40.7^{\circ} \mathrm{N}, 117.1^{\circ} \mathrm{E}\right)\end{array}$} & \multirow{3}{*}{$\begin{array}{c}\text { Jan. } 2005-\text { Dec. } \\
2008\end{array}$} & Spring & 43.2 & 46.0 & \multirow{3}{*}{$\mathrm{Xu}$ et al. (2011) } \\
\hline & & Summer & 46.7 & 47.2 & \\
\hline & & Fall & 29.8 & 36.8 & \\
\hline \multirow{6}{*}{$\begin{array}{c}\text { Xinglong } \\
\left(40.4^{\circ} \mathrm{N}, 117.6^{\circ} \mathrm{E}\right)\end{array}$} & \multirow{3}{*}{$\begin{array}{l}\text { Aug. } 2009- \\
\text { Jun. } 2010\end{array}$} & Spring & 52.3 & 45.3 & \multirow{3}{*}{ Wang et al. (2013) } \\
\hline & & Summer & 73 & 48.4 & \\
\hline & & Fall & 43 & 37.3 & \\
\hline & \multirow{3}{*}{$\begin{array}{c}\text { Jun. } 2005-\text { Sep. } \\
2006\end{array}$} & Spring & 52 & 45.3 & \multirow{3}{*}{ Ma et al. (2011) } \\
\hline & & Summer & 53 & 48.4 & \\
\hline & & Fall & 47 & 37.3 & \\
\hline \multirow{3}{*}{$\begin{array}{c}\text { Miyun } \\
\left(40.5^{\circ} \mathrm{N}, 116.8^{\circ} \mathrm{E}\right)\end{array}$} & \multirow{3}{*}{2006} & Spring & 48.7 & 45.5 & \multirow{3}{*}{ Wang et al. (2011) } \\
\hline & & Summer & 57.7 & 47.7 & \\
\hline & & Fall & 39.3 & 36.7 & \\
\hline $\begin{array}{c}\text { Wuqing } \\
\left(39.4^{\circ} \mathrm{N}, 117.0^{\circ} \mathrm{E}\right)\end{array}$ & $\begin{array}{c}\text { Jul. } 2009-\text { Sep. } \\
2009\end{array}$ & Summer & 44.1 & 50.9 & Han et al. (2013) \\
\hline \multirow{3}{*}{$\begin{array}{c}\text { Mountain Tai } \\
\left(36.3^{\circ} \mathrm{N}, 117.1^{\circ} \mathrm{E}\right)\end{array}$} & \multirow{3}{*}{$\begin{array}{l}\text { Mar. } 2004- \\
\text { Feb. } 2005\end{array}$} & Spring & 58.3 & 42.2 & \multirow{8}{*}{ Li et al. (2007) } \\
\hline & & Summer & 64.3 & 62.4 & \\
\hline & & Fall & 57.7 & 39.0 & \\
\hline \multirow{3}{*}{$\begin{array}{l}\text { Mountain Huang } \\
\left(30.1^{\circ} \mathrm{N}, 118.2^{\circ} \mathrm{E}\right)\end{array}$} & \multirow{3}{*}{$\begin{array}{l}\text { Mar. } 2004- \\
\text { Feb. } 2005\end{array}$} & Spring & 59 & 46.5 & \\
\hline & & Summer & 45.3 & 57.7 & \\
\hline & & Fall & 52.3 & 47.5 & \\
\hline \multirow{2}{*}{$\begin{array}{c}\text { Mountain Hua } \\
\left(34.5^{\circ} \mathrm{N}, 110.1^{\circ} \mathrm{E}\right)\end{array}$} & \multirow{2}{*}{$\begin{array}{l}\text { Mar. } 2004- \\
\text { Feb. } 2005\end{array}$} & Summer & 58.3 & 48.2 & \\
\hline & & Fall & 44.3 & 35.3 & \\
\hline \multirow{2}{*}{$\begin{array}{c}\text { Waliguan } \\
\left(36.3^{\circ} \mathrm{N}, 100.9^{\circ} \mathrm{E}\right) \\
\end{array}$} & Aug. 2006 & Summer & 58 & 49.2 & Xue et al. (2011) \\
\hline & Oct. 2011 & Fall & 53 & 43.7 & Wang et al. (2015) \\
\hline \multirow{3}{*}{$\begin{array}{c}\text { Dangxiong } \\
\left(30.5^{\circ} \mathrm{N}, 91.1^{\circ} \mathrm{E}\right)\end{array}$} & & Spring & 46 & 56.4 & \multirow{3}{*}{ Lin et al. (2015) } \\
\hline & $\begin{array}{l}\text { Sep. } 2009- \\
\text { Aug } 2011\end{array}$ & Summer & 37.7 & 46.0 & \\
\hline & Aug. 2011 & Fall & 31.3 & 49.2 & \\
\hline $\begin{array}{c}\text { Xinken } \\
\left(22.6^{\circ} \mathrm{N}, 113.6^{\circ} \mathrm{E}\right)\end{array}$ & Oct. 2004 & Fall & 49 & 54.4 & Zhang et al. (2008) \\
\hline & & Spring & 50.3 & 46.8 & \\
\hline Average & & Summer & 51.9 & 49.9 & \\
\hline & & Fall & 43.3 & 41.4 & \\
\hline
\end{tabular}


Table S5. Aerosol-induced changes in annual mean radiative and meteorological fields in eastern China ${ }^{\text {a }}$

\begin{tabular}{|c|c|c|c|c|c|c|}
\hline & \multicolumn{2}{|c|}{2010} & \multicolumn{2}{|c|}{2030 CLE } & \multicolumn{2}{|c|}{2030 MTFR } \\
\hline & No AIE & With AIE & No AIE & With AIE & No AIE & With AIE \\
\hline Temperature $\left({ }^{\circ} \mathrm{C}\right)$ & $\begin{array}{l}-\mathbf{0 . 6 3} \\
(0.36)\end{array}$ & $\begin{array}{l}-\mathbf{0 . 7 5} \\
(0.48)\end{array}$ & $\begin{array}{l}-0.36^{*} \\
(0.59)\end{array}$ & $\begin{array}{l}\mathbf{- 0 . 5 2} \\
(0.53)\end{array}$ & $\begin{array}{l}-0.31^{*} \\
(0.51)\end{array}$ & $\begin{array}{l}-0.08 \\
(0.35)\end{array}$ \\
\hline Direct SW $\left(\mathrm{W} \mathrm{m}^{-2}\right)$ & $\begin{array}{c}\mathbf{- 3 5 . 0} \\
(3.8)\end{array}$ & $\begin{array}{c}\mathbf{- 3 3 . 4} \\
(3.0)\end{array}$ & $\begin{array}{c}-33.2^{*} \\
(3.2)\end{array}$ & $\begin{array}{c}-33.6^{*} \\
(4.1)\end{array}$ & $\begin{array}{c}-15.1^{*} \\
(3.6)\end{array}$ & $\begin{array}{c}-15.9^{*} \\
(3.0)\end{array}$ \\
\hline Diffuse SW $\left(\mathrm{W} \mathrm{m}^{-2}\right)$ & $\begin{array}{c}\mathbf{8 . 8}^{*} \\
(1.7)\end{array}$ & $\begin{array}{l}3.0^{*} \\
(1.7)\end{array}$ & $\begin{array}{l}11.0^{*} \\
(2.3)\end{array}$ & $\begin{array}{l}\mathbf{5 . 6} \\
(2.4)\end{array}$ & $\begin{array}{l}6.5^{*} \\
(1.7)\end{array}$ & $\begin{array}{l}2.0^{*} \\
(1.7)\end{array}$ \\
\hline RH (\%) & $\begin{array}{l}1.6^{*} \\
(1.7)\end{array}$ & $\begin{array}{c}0.6 \\
(1.2)\end{array}$ & $\begin{array}{l}1.1^{*} \\
(1.9)\end{array}$ & $\begin{array}{l}1.0 \\
(2.5)\end{array}$ & $\begin{array}{c}0.7 \\
(1.9)\end{array}$ & $\begin{array}{l}1.6^{*} \\
(2.3)\end{array}$ \\
\hline Precipitation $\left(\mathrm{mm} \mathrm{d}^{-1}\right)$ & $\begin{array}{c}0.05 \\
(0.33)\end{array}$ & $\begin{array}{l}-\mathbf{0 . 4 8} \\
(0.39)\end{array}$ & $\begin{array}{l}-0.07 \\
(0.51)\end{array}$ & $\begin{array}{l}-\mathbf{0 . 3 4} \\
(0.40)\end{array}$ & $\begin{array}{c}0.10 \\
(0.46)\end{array}$ & $\begin{array}{c}0.01 \\
(0.37)\end{array}$ \\
\hline Middle cloud (\%) & $\begin{array}{c}0.8^{*} \\
(1.2)\end{array}$ & $\begin{array}{c}0.1 \\
(1.4)\end{array}$ & $\begin{array}{c}0.2 \\
(1.3)\end{array}$ & $\begin{array}{l}-0.1 \\
(1.7)\end{array}$ & $\begin{array}{c}0.6 \\
(1.4)\end{array}$ & $\begin{array}{c}0.0 \\
(0.8)\end{array}$ \\
\hline Evaporation $(\%)^{b}$ & $\begin{array}{l}\mathbf{- 4 . 0} \\
(1.4)\end{array}$ & $\begin{array}{l}\mathbf{- 8 . 3} \\
(1.9)\end{array}$ & $\begin{array}{l}\mathbf{- 2 . 8} \\
(2.2)\end{array}$ & $\begin{array}{l}-7.0^{*} \\
(1.5)\end{array}$ & $\begin{array}{l}-0.6 \\
(2.7)\end{array}$ & $\begin{array}{l}\mathbf{- 2 . 6} \\
(1.2)\end{array}$ \\
\hline Soil moisture $(\%)^{b}$ & $\begin{array}{c}7.0 * \\
(7.4)\end{array}$ & $\begin{array}{l}-0.2 \\
(9.5)\end{array}$ & $\begin{array}{c}3.7 \\
(8.9)\end{array}$ & $\begin{array}{c}1.4 \\
(9.5)\end{array}$ & $\begin{array}{l}5.2^{*} \\
(9.2)\end{array}$ & $\begin{array}{c}4.8 \\
(7.3)\end{array}$ \\
\hline
\end{tabular}

a Significant changes $(p<0.05)$ are marked with asterisks. Significant changes $(p<0.01)$ are shown in bold. Values refer to mean changes and one standard deviation (bracketed). Abbreviations for variables include shortwave radiation (SW), relative humidity $(\mathrm{RH})$, and aerosol indirect radiative effects (AIE). All the values are the averages over the box domain of Fig. S5a.

${ }^{\mathrm{b}}$ Changes are relative values: $(\mathrm{Y} 2-\mathrm{Y} 1) / \mathrm{Y} 1 \times 100 \%$, where $\mathrm{Y} 2$ and $\mathrm{Y} 1$ are, respectively, simulations with and without aerosol effects. 
Table S6. Aerosol-induced changes in summer mean radiative and meteorological fields in eastern China ${ }^{\text {a }}$

\begin{tabular}{|c|c|c|c|c|c|c|}
\hline & \multicolumn{2}{|c|}{2010} & \multicolumn{2}{|c|}{2030 CLE } & \multicolumn{2}{|c|}{2030 MTFR } \\
\hline & No AIE & With AIE & No AIE & With AIE & No AIE & With AIE \\
\hline Temperature $\left({ }^{\circ} \mathrm{C}\right)$ & $\begin{array}{c}\mathbf{- 0 . 4 8}^{*} \\
(0.37)\end{array}$ & $\begin{array}{l}-\mathbf{0 . 6 9} \\
(0.36)\end{array}$ & $\begin{array}{l}\mathbf{- 0 . 3 4} \\
(0.49)\end{array}$ & $\begin{array}{l}-\mathbf{0 . 5 3} \\
(0.45)\end{array}$ & $\begin{array}{l}-0.31^{*} \\
(0.39)\end{array}$ & $\begin{array}{l}-0.32 \\
(0.50)\end{array}$ \\
\hline Direct SW $\left(\mathrm{W} \mathrm{m}^{-2}\right)$ & $\begin{array}{r}-35.9^{*} \\
(5.3)\end{array}$ & $\begin{array}{c}-35.4^{*} \\
(4.6)\end{array}$ & $\begin{array}{c}-36.4^{*} \\
(3.7)\end{array}$ & $\begin{array}{c}\mathbf{- 3 8 . 8} \\
(6.0)\end{array}$ & $\begin{array}{c}-17.4^{*} \\
(3.4)\end{array}$ & $\begin{array}{c}-16.6 * \\
(5.3)\end{array}$ \\
\hline Diffuse SW $\left(\mathrm{W} \mathrm{m}^{-2}\right)$ & $\begin{array}{l}11.7^{*} \\
(3.0)\end{array}$ & $\begin{array}{c}6.2^{*} \\
(3.0)\end{array}$ & $\begin{array}{l}13.2^{*} \\
(2.7)\end{array}$ & $\begin{array}{l}7.0^{*} \\
(1.8)\end{array}$ & $\begin{array}{l}7.4^{*} \\
(3.3)\end{array}$ & $\begin{array}{l}3.1^{*} \\
(1.6)\end{array}$ \\
\hline RH (\%) & $\begin{array}{l}1.7^{*} \\
(2.8)\end{array}$ & $\begin{array}{c}0.0 \\
(2.6)\end{array}$ & $\begin{array}{l}2.2^{*} \\
(2.5)\end{array}$ & $\begin{array}{c}2.7^{*} \\
(1.8)\end{array}$ & $\begin{array}{l}1.9^{*} \\
(2.3)\end{array}$ & $\begin{array}{c}1.5 \\
(2.2)\end{array}$ \\
\hline Precipitation $\left(\mathrm{mm} \mathrm{d}^{-1}\right)$ & $\begin{array}{c}0.15 \\
(0.96)\end{array}$ & $\begin{array}{l}-\mathbf{0 . 8 5} \\
(0.79)\end{array}$ & $\begin{array}{c}0.11 \\
(0.88)\end{array}$ & $\begin{array}{c}0.05 \\
(0.82)\end{array}$ & $\begin{array}{c}0.40 \\
(0.72)\end{array}$ & $\begin{array}{c}0.17 \\
(0.58)\end{array}$ \\
\hline Middle cloud (\%) & $\begin{array}{c}1.0 \\
(2.1)\end{array}$ & $\begin{array}{c}0.3 \\
(1.7)\end{array}$ & $\begin{array}{c}0.4 \\
(2.1)\end{array}$ & $\begin{array}{c}0.6 \\
(2.1)\end{array}$ & $\begin{array}{c}0.6 \\
(2.1)\end{array}$ & $\begin{array}{l}-0.1 \\
(2.3)\end{array}$ \\
\hline Evaporation (\%) & $\begin{array}{l}\mathbf{- 3 . 2} \\
(2.6)\end{array}$ & $\begin{array}{l}\mathbf{- 8 . 0 *} \\
(2.4)\end{array}$ & $\begin{array}{l}-3.2^{*} \\
(3.6)\end{array}$ & $\begin{array}{l}-7.7^{*} \\
(2.7)\end{array}$ & $\begin{array}{l}-0.7 \\
(3.8)\end{array}$ & $\begin{array}{l}\mathbf{- 2 . 5} \\
(2.9)\end{array}$ \\
\hline Soil moisture (\%) & $\begin{array}{c}7.6^{*} \\
(13.2)\end{array}$ & $\begin{array}{c}-2.5 \\
(12.0)\end{array}$ & $\begin{array}{c}5.7^{*} \\
(11.2)\end{array}$ & $\begin{array}{c}6.7 \\
(11.0)\end{array}$ & $\begin{array}{l}\mathbf{8 . 1}^{*} \\
(9.2)\end{array}$ & $\begin{array}{c}3.9 \\
(9.5)\end{array}$ \\
\hline
\end{tabular}

\footnotetext{
${ }^{a}$ The same as Table S5 but for summer variables
} 
Tundra

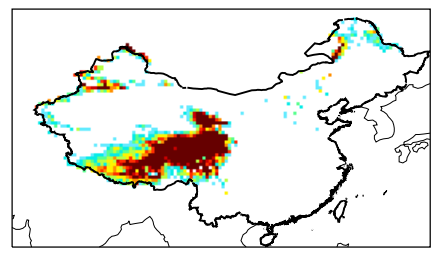

Deciduous broadleaf forest

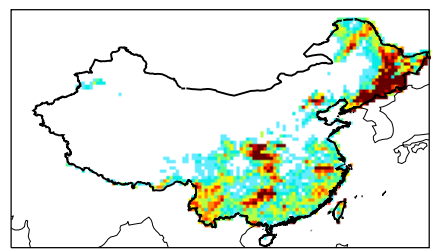

C3 cropland

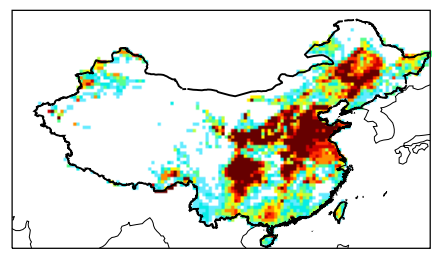

C3 grassland

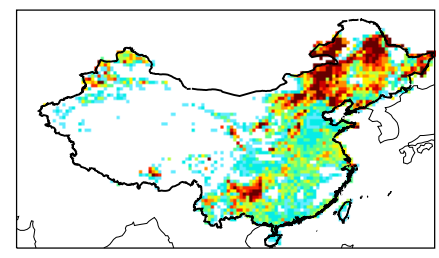

Everereen needleleaf forest

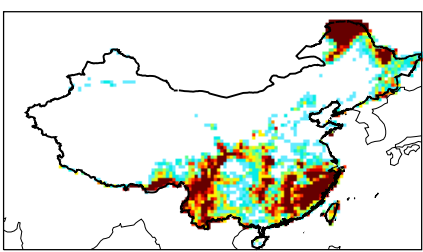

C4 grassland

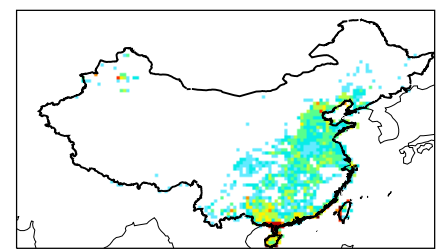

Shrubland

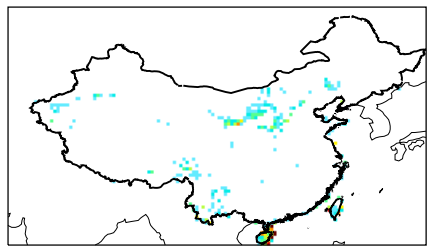

Tropical rainforest

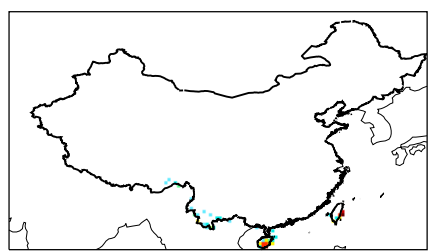

Non-vegetated

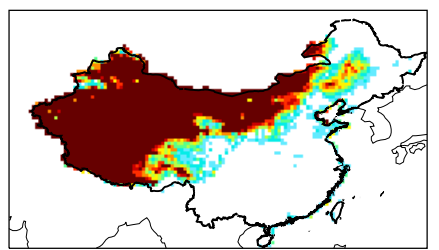

$50(\%)$

Figure S1. Land cover fraction in China. Each grid square contains vegetation cover for 8 plant functional types (PFTs) used by the YIBs vegetation model. 

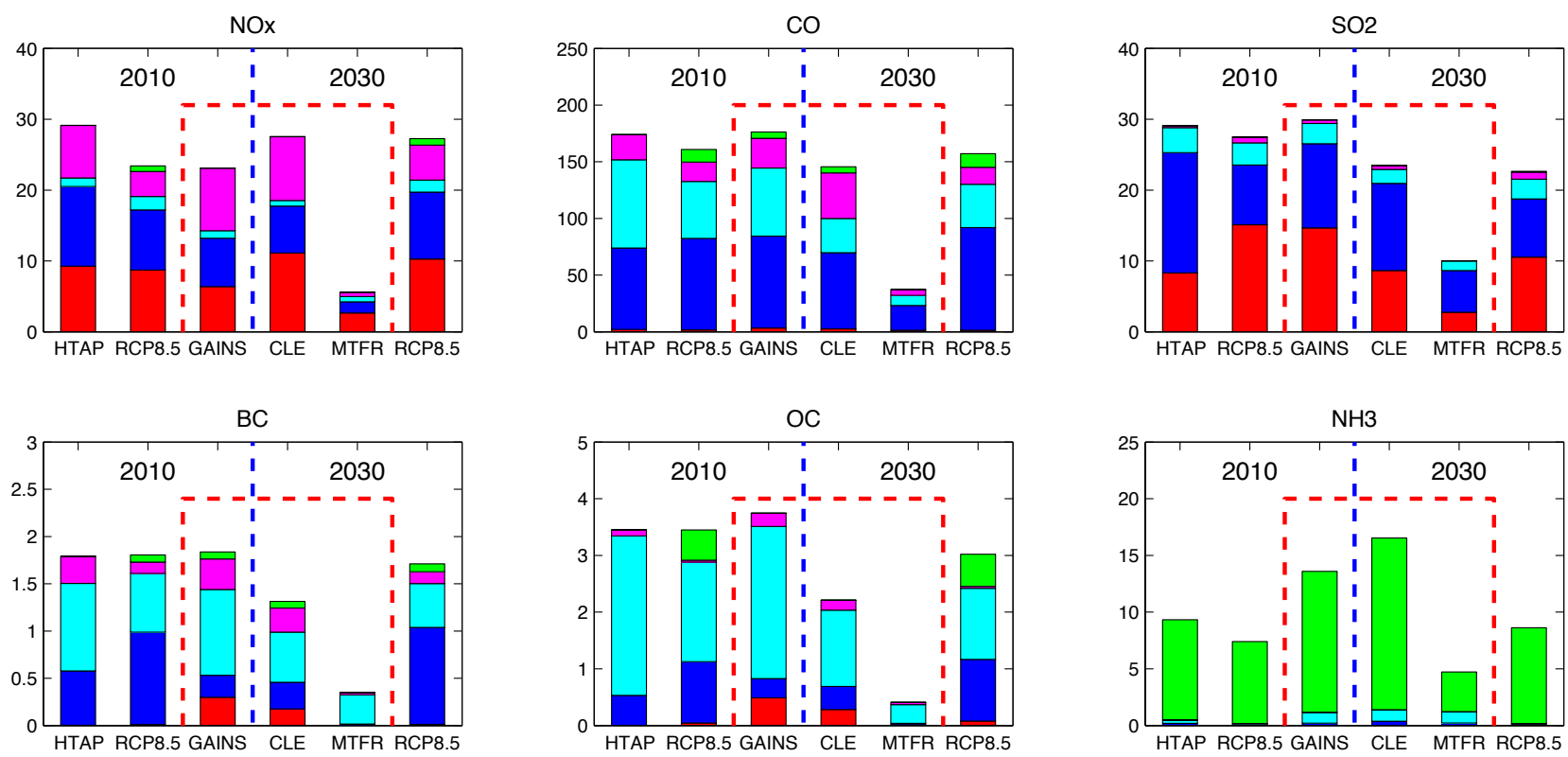

ENERGY

INDUSTRY

RESIDENTIAL

TRANSPORT

AGRICULTURE

Figure S2. Chinese emissions from global inventories used for simulations and intercomparison. Three inventories, GAINS for 2010, GAINS_CLE for 2030, and GAINS_MTFR for 2030, within the red dashed boxes are used in the simulations. The other three, HTAP for 2010, RCP8.5 for 2010, and RCP8.5 for 2030 are used for comparison. All emissions are broken into five sectors, including energy, industry, residential, transportation, and agriculture. The inventory GAINS refers to the v4a version of the Greenhouse Gas and Air Pollution Interactions and Synergies integrated assessment model (http://gains.iiasa.ac.at/models/). The inventory GAINS_CLE is developed based on current legislation emissions. The inventory GAINS_MTFR is developed based on the maximum technically feasible reductions. The inventory HTAP is adopted from the Emissions Database for Global Atmospheric Research (EDGAR,

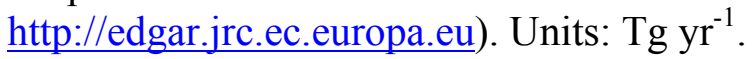



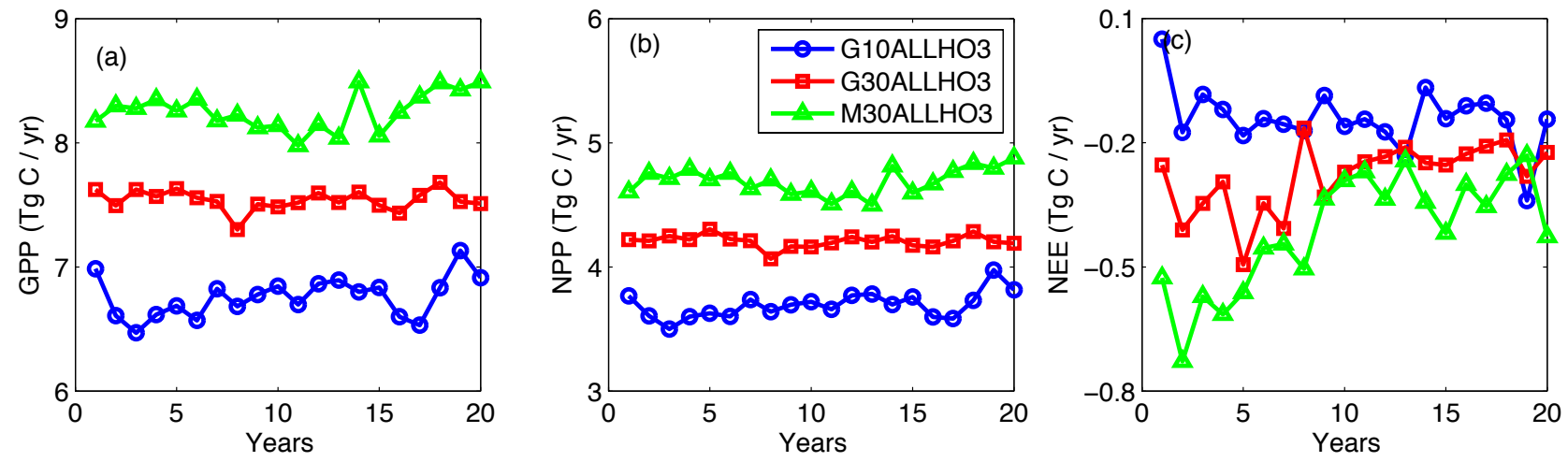

Figure S3. Simulated year-to-year carbon fluxes. Annual total carbon fluxes of (a) GPP, (b) NPP, and (c) NEE in China for the 20-year simulations with ModelE2-YIBs climate model (Table 2). Different colors represent results from simulations G10ALLHO3 (blue), G30ALLHO3 (red), and M30ALLHO3 (green), indicating different influences from $\mathrm{CO}_{2}$ fertilization, climate change, and $\mathrm{O}_{3}$ damages. 
(a) Simulated annual GPP

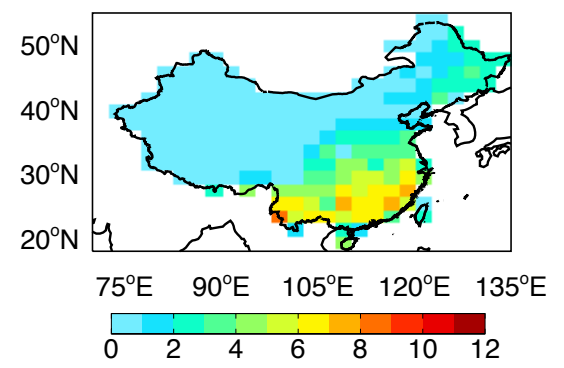

(d) Simulated annual NPP

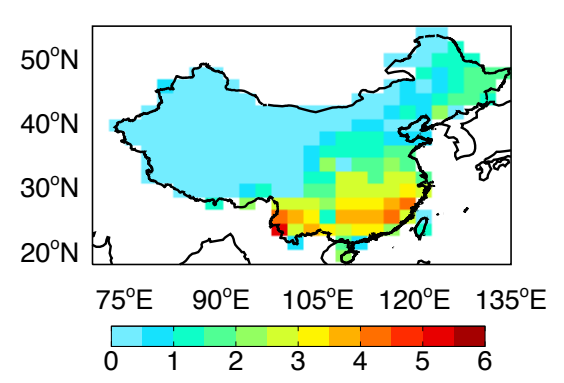

(b) Observed annual GPP

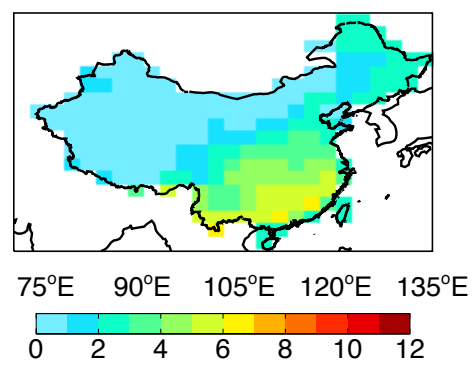

(e) Observed annual NPP

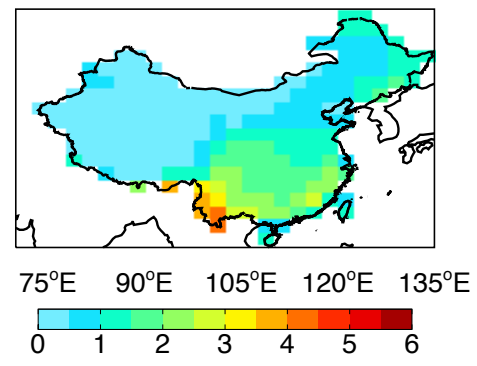

(c) GPP

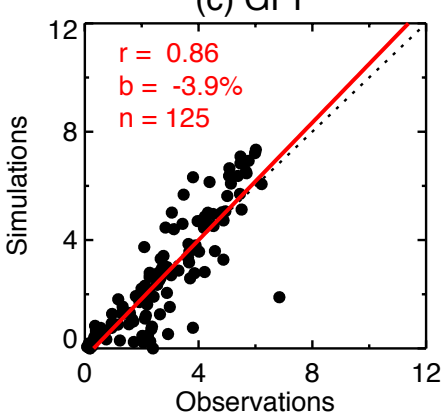

(f) NPP

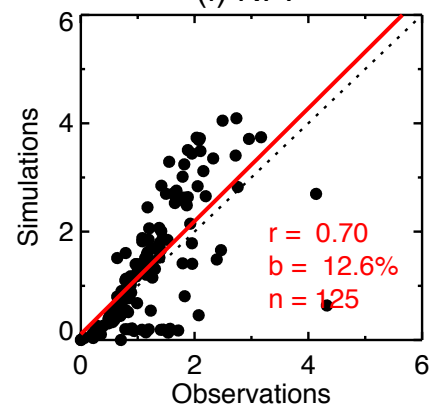

Figure S4. Similar to Fig. 1 but for the annual carbon fluxes. 
(a) Simulated annual AOD

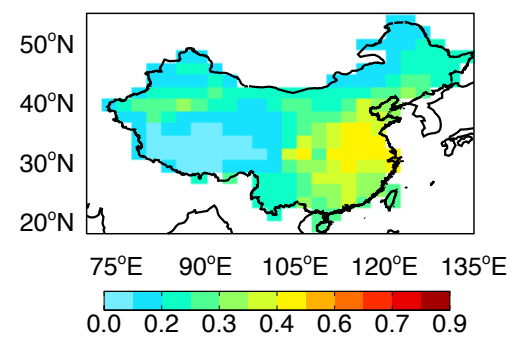

(d) Simulated annual [O3]

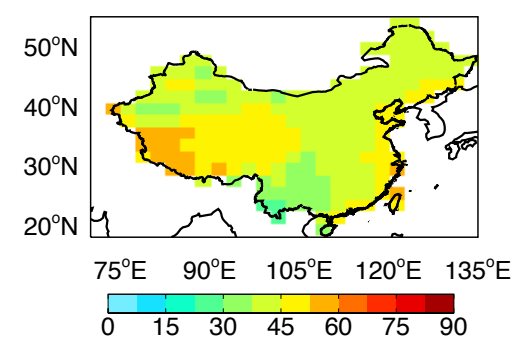

(g) Simulated annual PM2.5

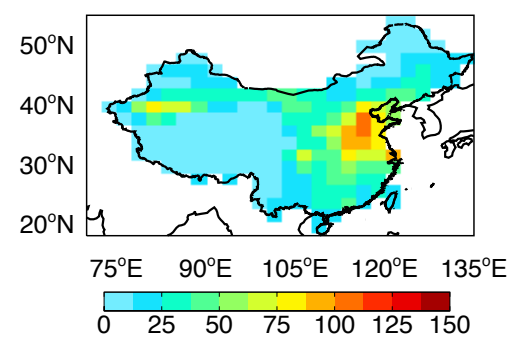

(b) Observed annual AOD

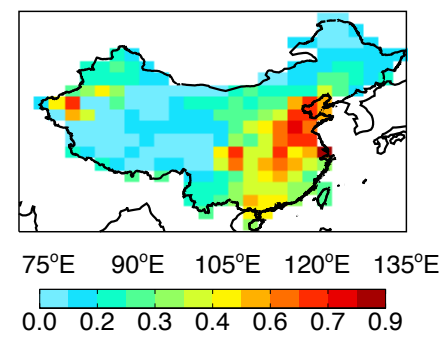

(e) Observed annual [O3]

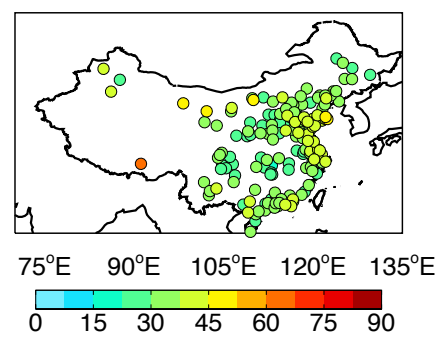

(h) Observed annual PM2.5

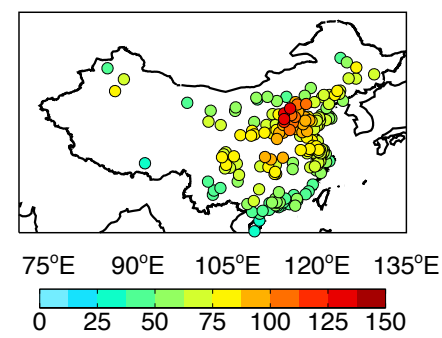

(c) $\mathrm{AOD}$
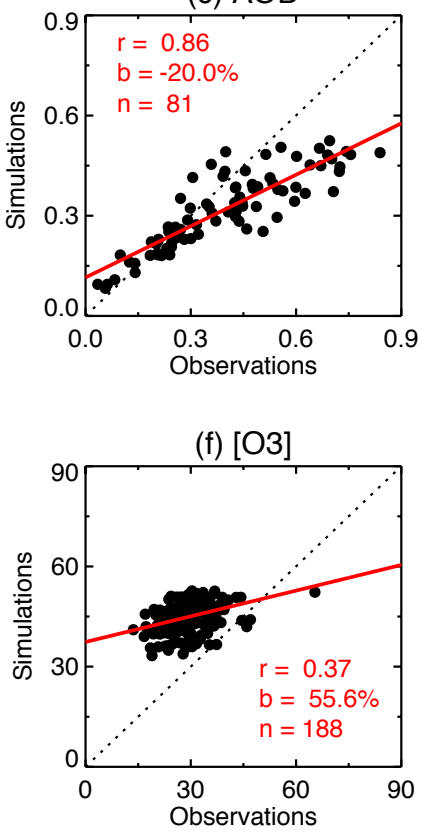

(i) PM2.5

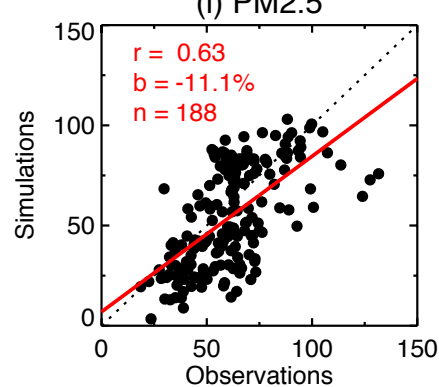

Figure S5. Similar to Fig. 2 but for the annual air pollution. 
(a) Simulated annual total SW

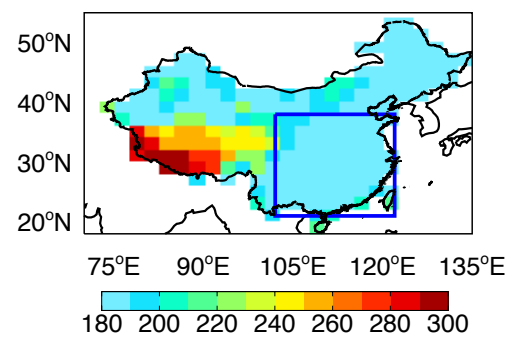

(d) Simulated annual diffuse fraction

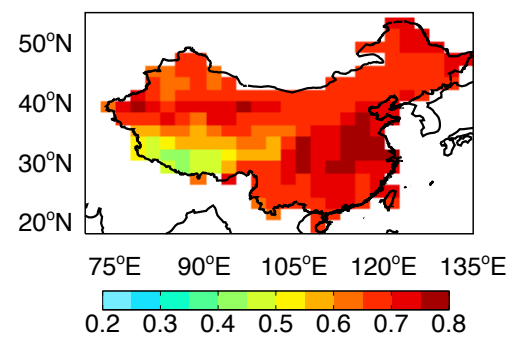

(b) Observed annual total SW

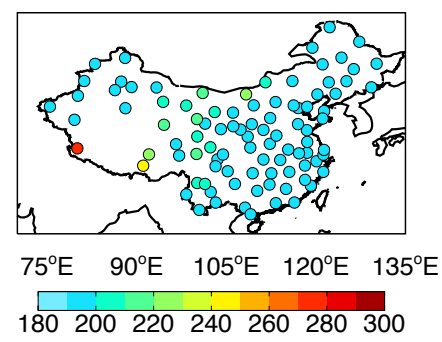

(e) Observed annual diffuse fraction

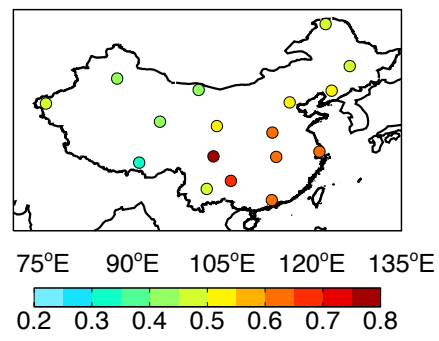

(c) total SW
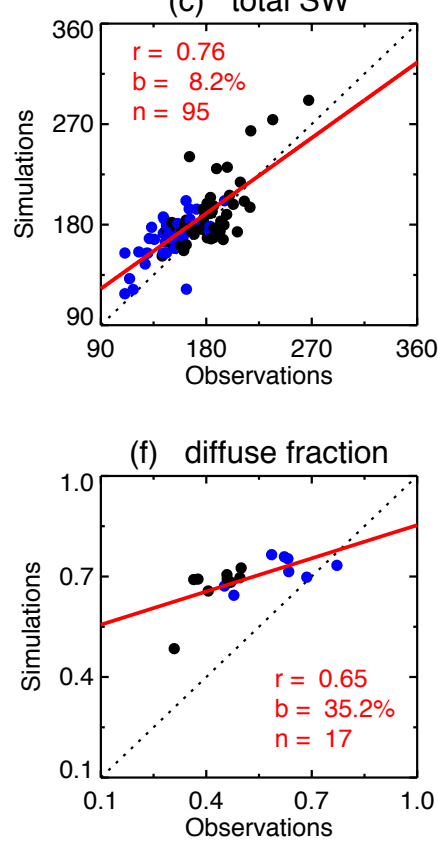

Figure S6. Similar to Fig. 4 but for the annual radiative fluxes. 
(a) Summer AOD ( 0.3 )

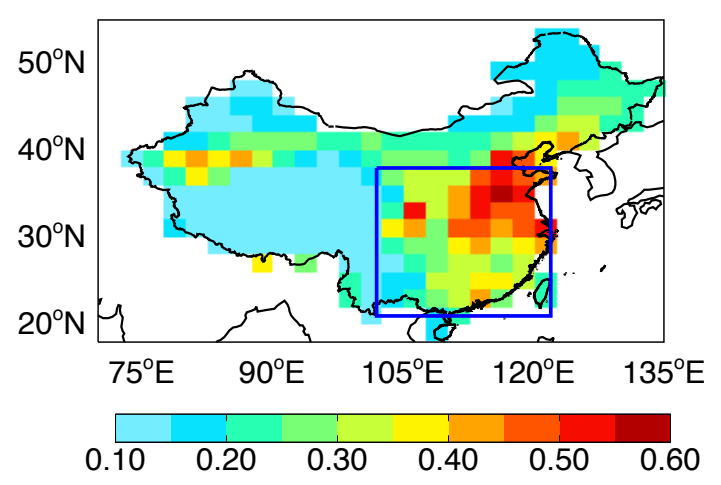

(c) Summer [O3] ( $47 \mathrm{ppbv}$ )

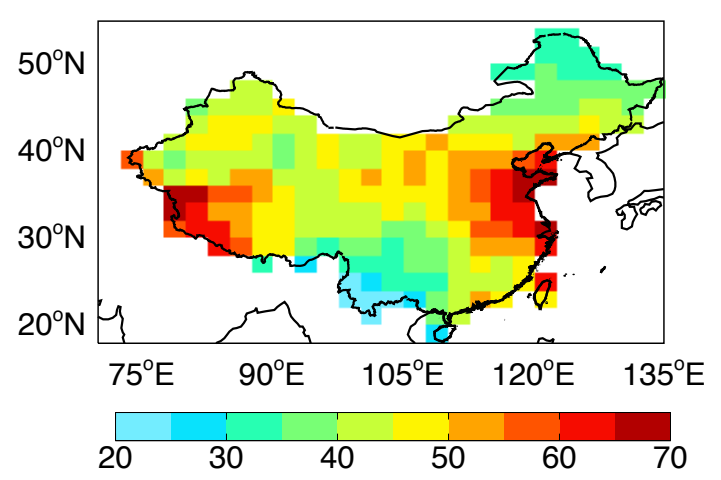

(e) Summer PM2.5 ( $\left.33 \mu \mathrm{g} \mathrm{m}^{-3}\right)$

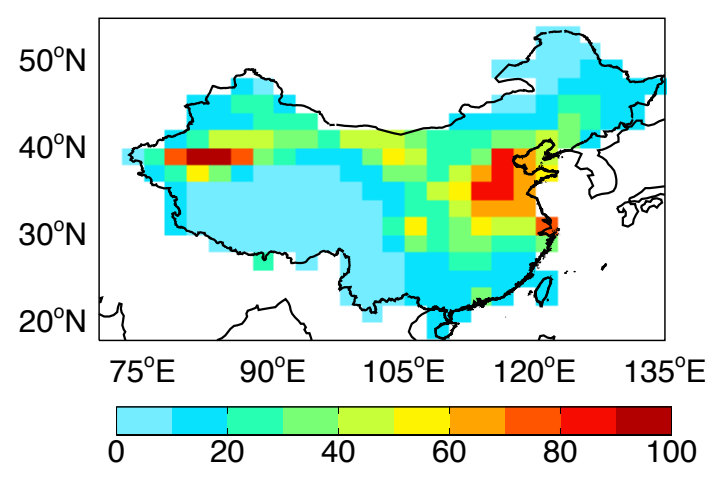

(b) Anth. percentage in AOD ( $79 \%)$

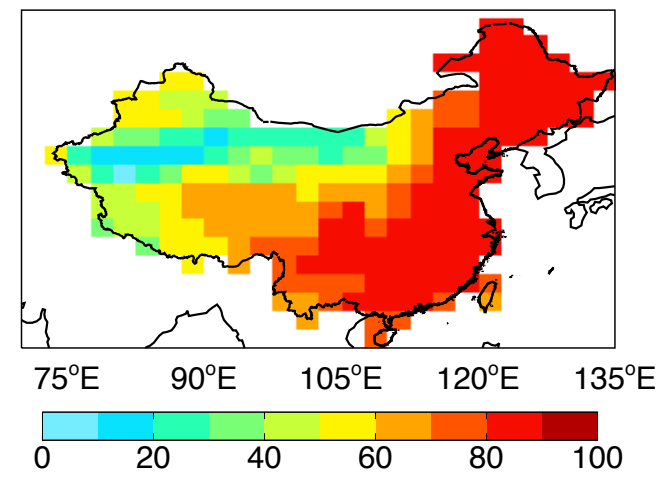

(d) Anth. percentage in [O3] ( $76 \%)$

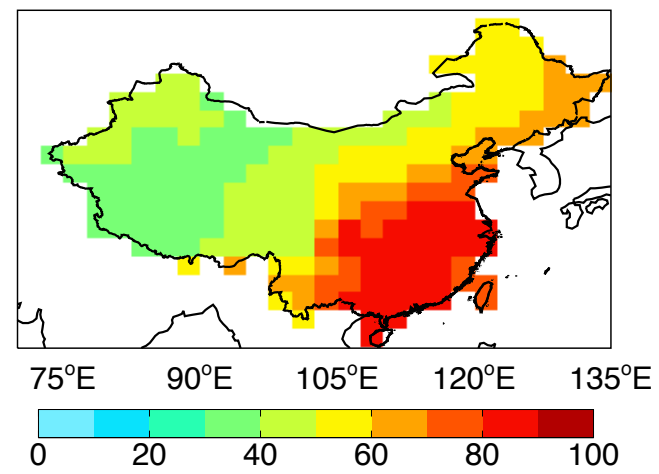

(f) Anth. percentage in PM2.5 ( $76 \%$ )

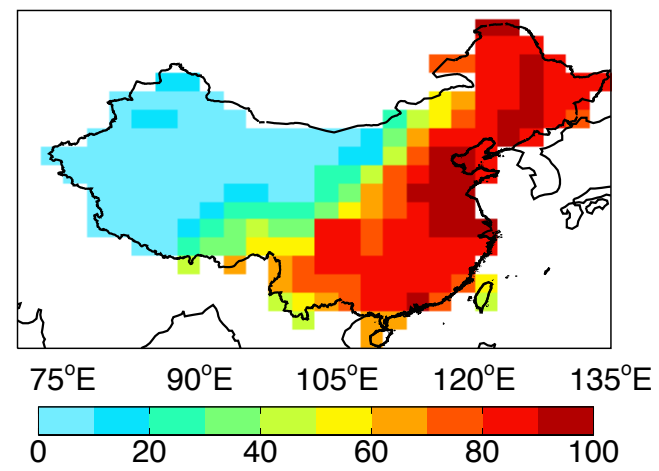

Figure S7. Simulated air pollution in 2010 and the contributions from anthropogenic emissions. Panels shown are for $(\mathrm{a}, \mathrm{b}) \mathrm{AOD}$ at $550 \mathrm{~nm},(\mathrm{c}, \mathrm{d})\left[\mathrm{O}_{3}\right]$, and (e, f) $\mathrm{PM}_{2.5}$ concentrations for the summer of the year 2010. Results for the left panels are from G10ALLNO3. Results for the right panels are calculated as (1 G10NATNO3/G10ALLNO3) $\times 100 \%$. The average value over the box domain $\left(21^{\circ}-\right.$ $\left.38^{\circ} \mathrm{N}, 102^{\circ}-122^{\circ} \mathrm{E}\right)$ of (a) is shown in the title bracket of each subpanel. 
(a) Relative changes in stomatal conductance (\%)

(b) Absolute changes in temperature (K)
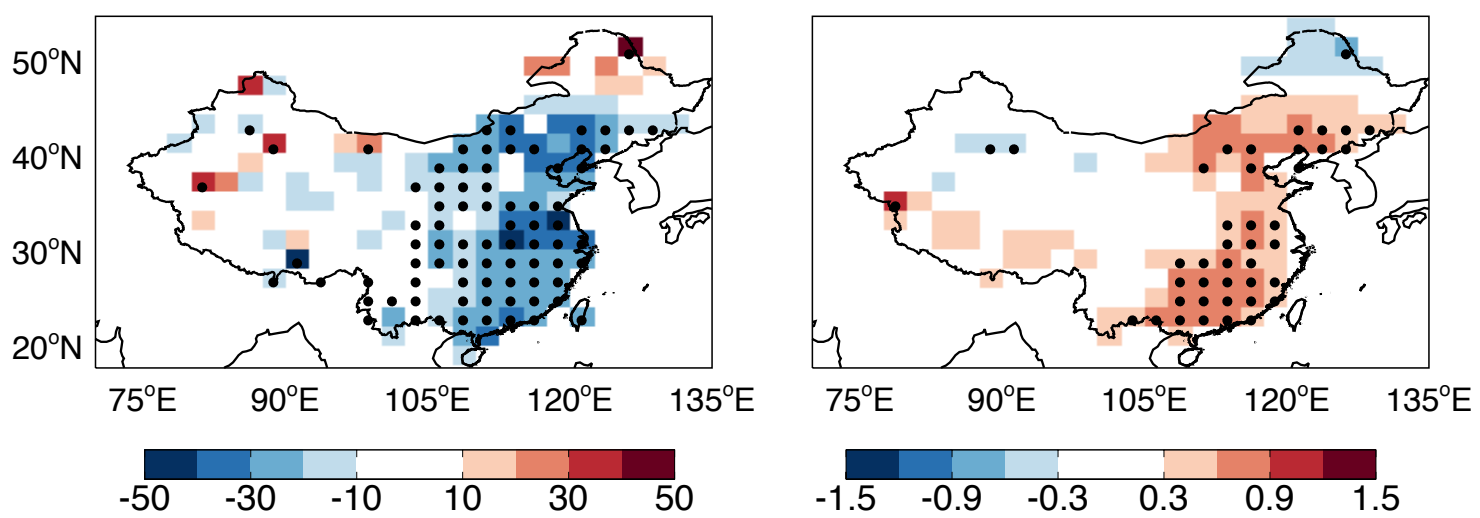

(c) Absolute changes in relative humdiity (\%)

(d) Absolute changes in precipitation $\left(\mathrm{mm}\right.$ day $\left.^{-1}\right)$
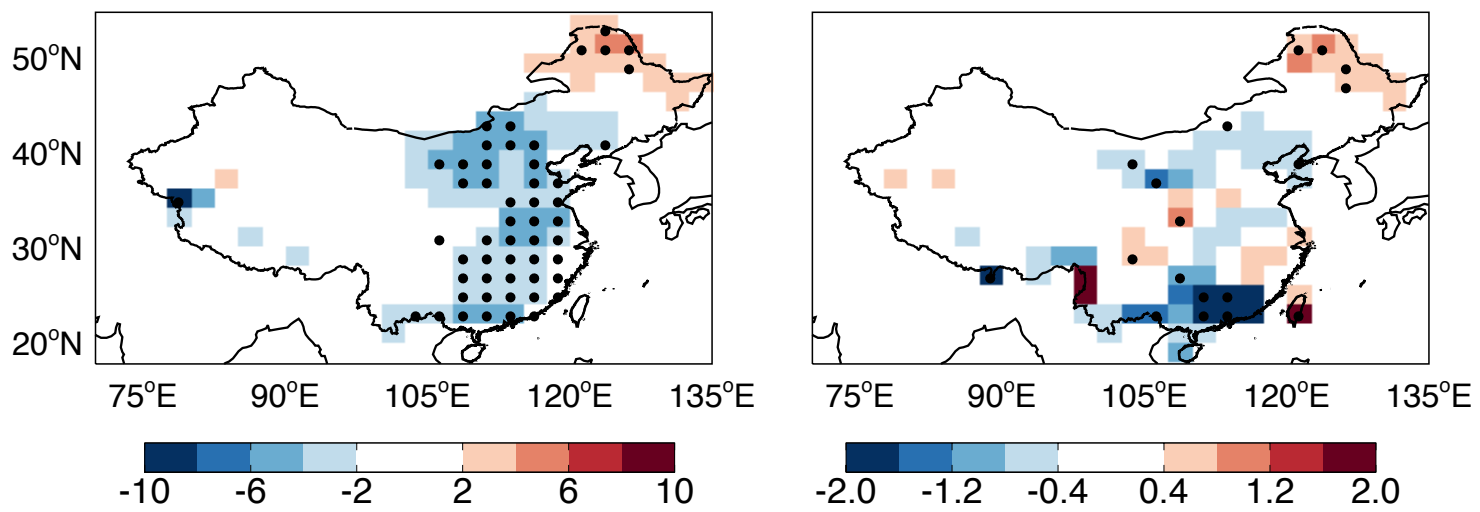

Figure S8. Changes in (a) stomatal conductance and the consequent changes in (b) temperature, (c) relative humidity, and (d) precipitation due to $\mathrm{O}_{3}$ vegetation damages. The relative changes in (a) are calculated as ( $\frac{1}{2}($ G10ALLHO3+G10ALLLO3)/ G10ALLNO3 - 1) $\times 100 \%$. The absolute changes in (b-d) are calculated as $\frac{1}{2}($ G10ALLHO3+G10ALLLO3 $)-$ G10ALLNO3. Significant changes $(p<0.05)$ are marked with black dots. 
(a) $\triangle$ GPP by NAT O3 low $(-0.1 \%)$

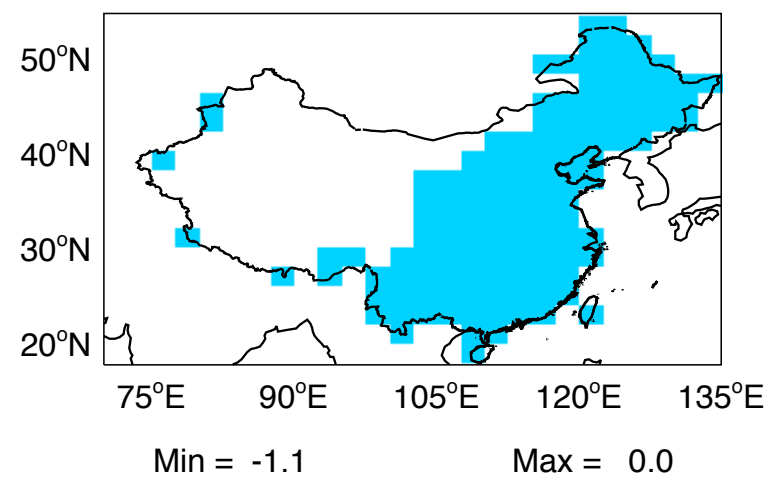

(c) $\triangle$ GPP by ALL O3 low ( $-3.4 \%)$

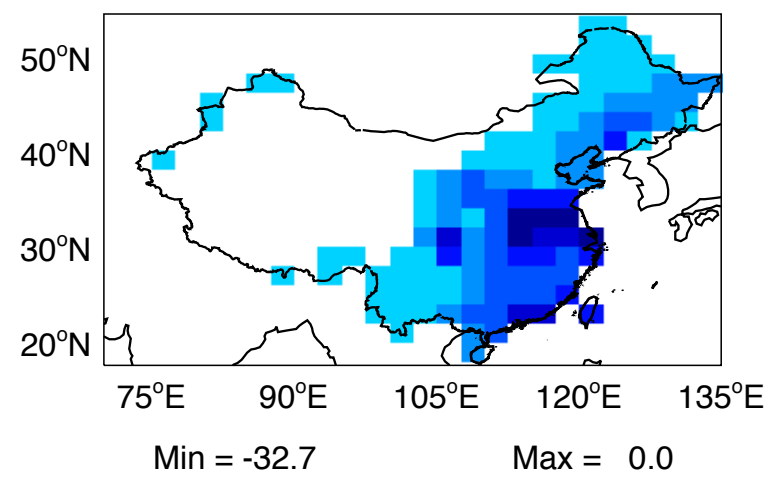

(b) $\triangle$ GPP by NAT O3 high ( $-0.2 \%)$

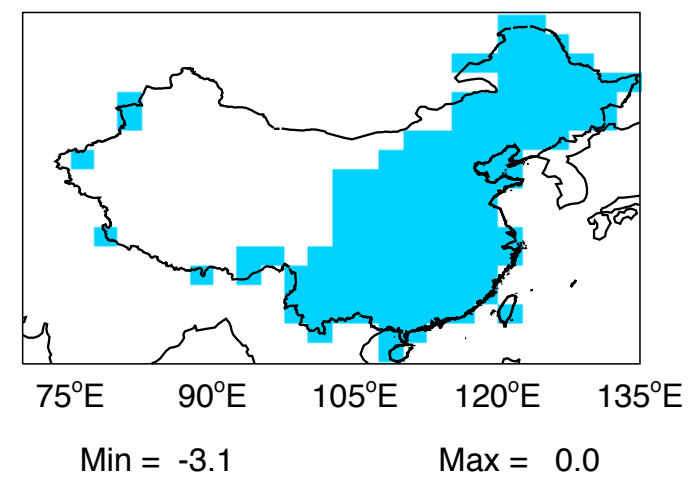

(d) $\triangle$ GPP by ALL O3 high ( $-6.6 \%)$

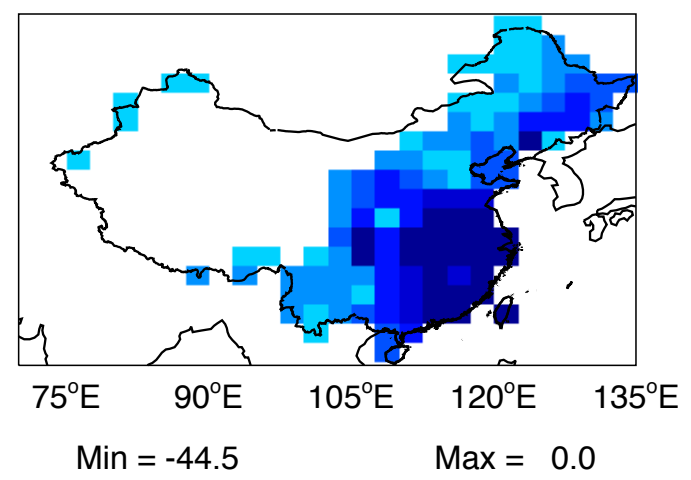

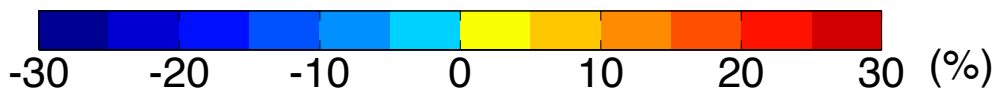

Figure S9. Predicted offline percentage damage to summer GPP caused by $\mathrm{O}_{3}(\mathrm{a}, \mathrm{b})$ with natural emission alone and (c, d) with both natural and anthropogenic emissions. Simulations are performed with the climate model ModelE2-YIBs, which does not feed $\mathrm{O}_{3}$ vegetation damages back to affect biometeorology, plant growth, and ecosystem physiology. Results are calculated as (a) (G10NATLO3_OFF/G10NATNO3 - 1) $\times 100 \%$, (b) (G10NATHO3_OFF/G10NATNO3 - 1) $\times 100 \%$, (c) (G10ALLLO3_OFF $/$ G10ALLNO3 - 1) $\times 100 \%$, and (d) (G10ALLHO3 OFF /G10ALLNO3 - 1) $\times 100 \%$. The maximum and minimum fractional changes are marked at the bottom of each panel. The average value over the whole China domain is shown in the title bracket of each subpanel. 
(a) Summer total SW (230.1)

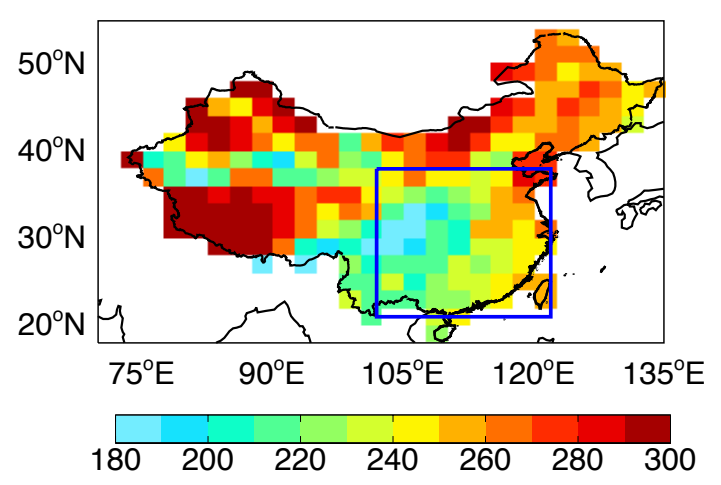

(c) Summer direct SW ( 98.8 )

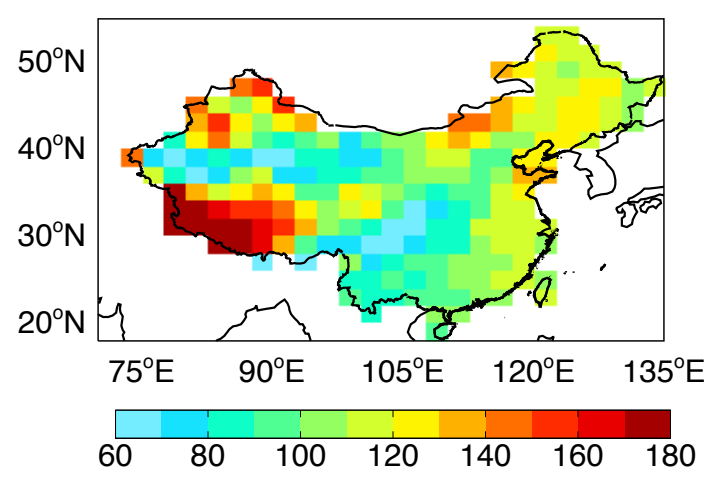

(e) Summer diffuse SW (131.3)

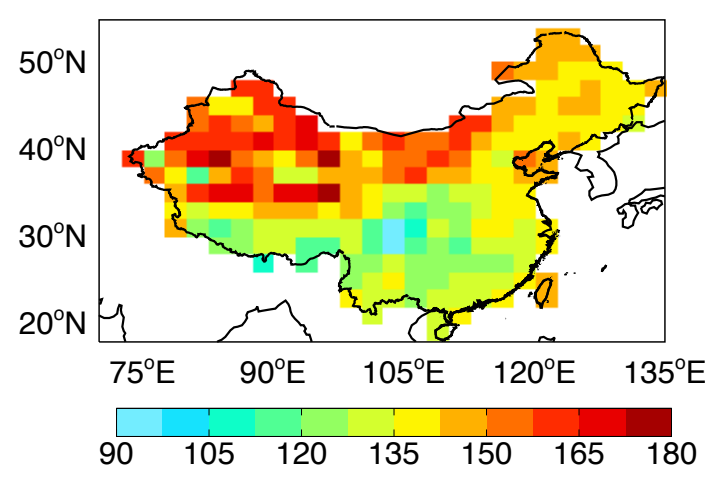

(b) Summer changes in total SW (-24.3)

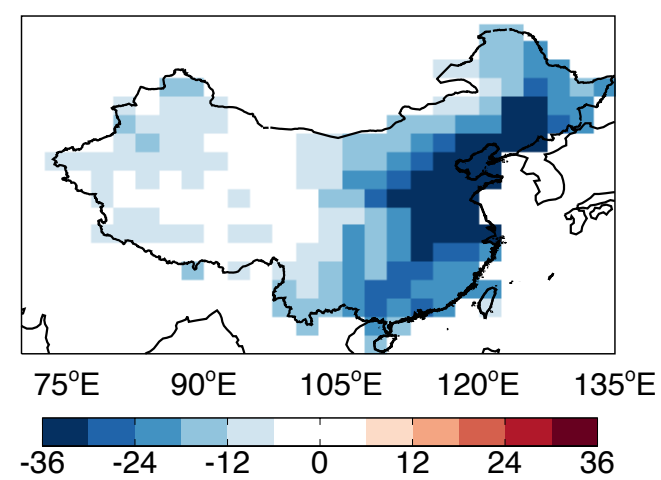

(d) Summer changes in direct SW (-35.9)

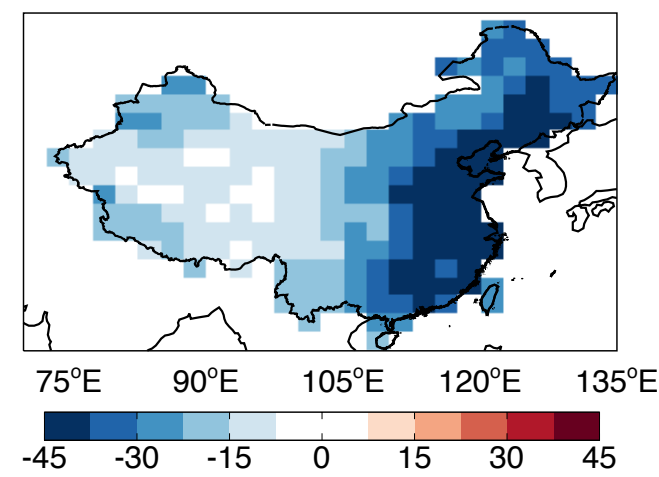

(f) Summer changes in diffuse SW (11.7)

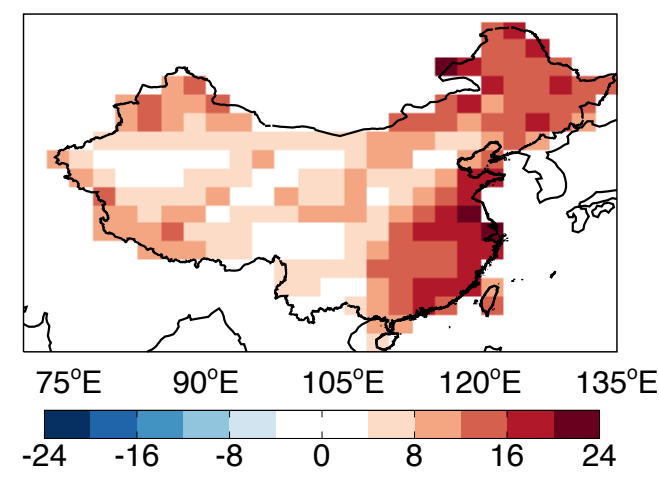

Figure S10. Simulated summer surface radiation and the perturbations by anthropogenic aerosols. Simulations are performed with the climate model ModelE2-YIBs. Panels show (a) total, (c) direct, and (e) diffuse visible solar radiation without anthropogenic aerosols (G10NATNO3) and (b, d, f) their perturbations by anthropogenic aerosols (G10ALLNO3 - G10NATNO3) at the year 2010. The average value over the box domain of (a) is shown in the top center of each subpanel. Units: $\mathrm{W} \mathrm{m}^{-2}$. 
(a) Absolute changes in temperature $(\mathrm{K})$

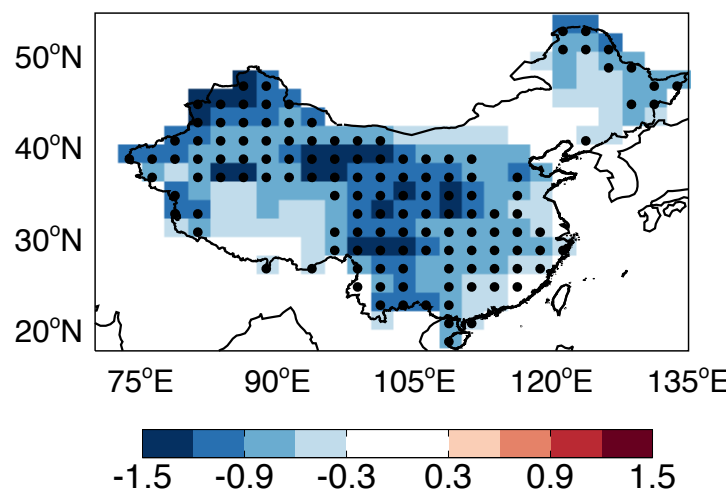

(c) Absolute changes in $\mathrm{RH}(\%)$

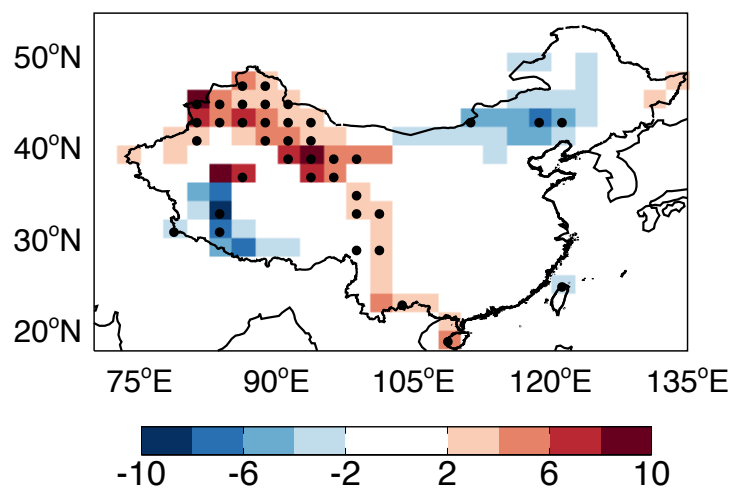

(e) Relative changes in evaporation (\%)

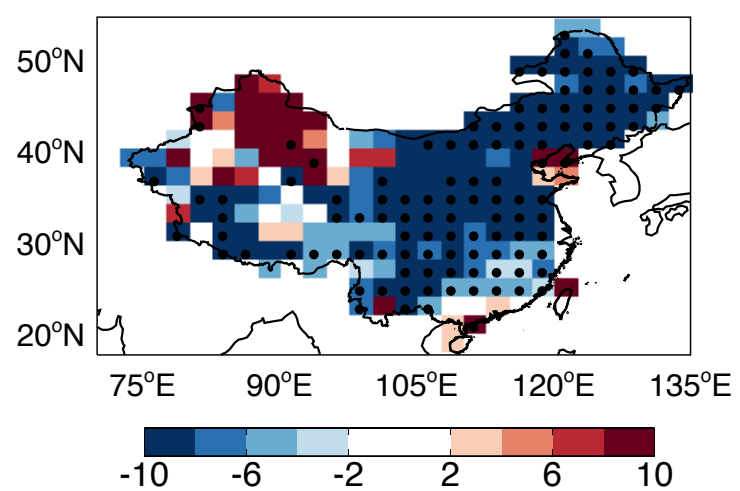

(b) Absolute changes in precipitation ( $\mathrm{mm}$ day $\left.^{-1}\right)$

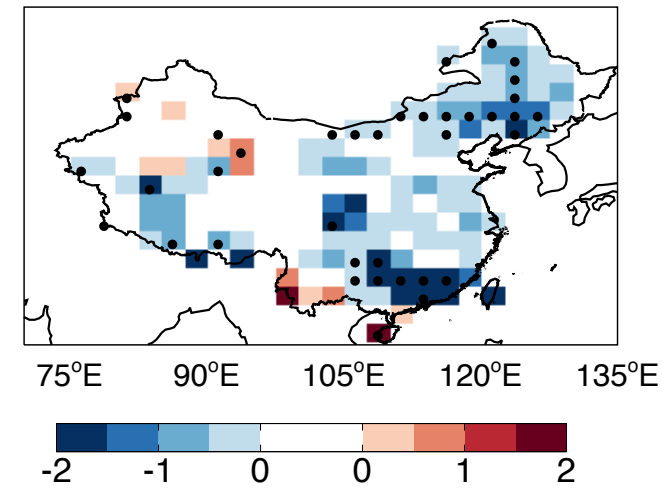

(d) Relative changes in middle cloud (\%)

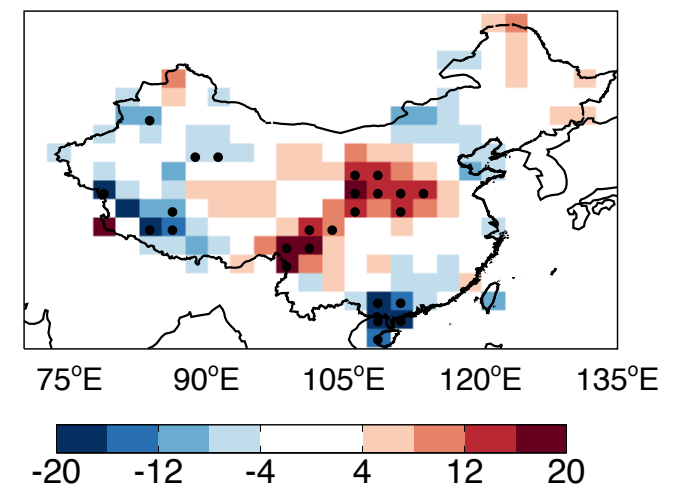

(f) Relative changes in soil water (\%)

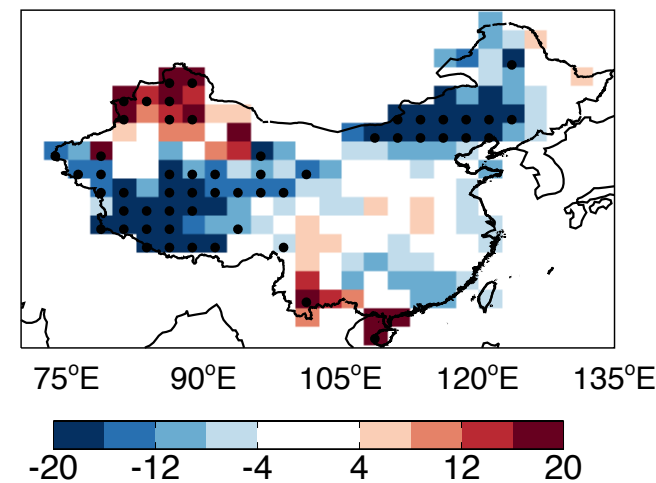

Figure S11. Changes in summer meteorology due to both direct and indirect radiative effects of anthropogenic aerosols. All changes are calculated as the differences between the simulations G10ALLNO3_AIE and G10NATNO3_AIE. For (a) temperature, (b) precipitation, and (c) relative humidity, we show the absolute changes as G10ALLNO3_AIE - G10NATNO3_AIE. For (d) middle cloud cover, (e) evaporation, and (f) soil water content, we show the relative changes as (G10ALLNO3_AIE/G10NATNO3_AIE -1$) \times 100 \%$. Significant changes $(p<0.05)$ are marked with black dots. 
(a) $\triangle$ NPP by ALL changes $(-0.05)$

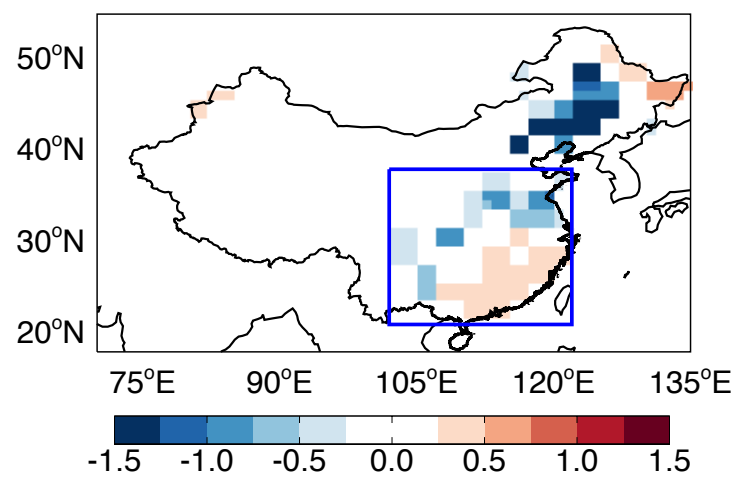

(c) $\triangle$ NPP by PAR changes $(0.01)$

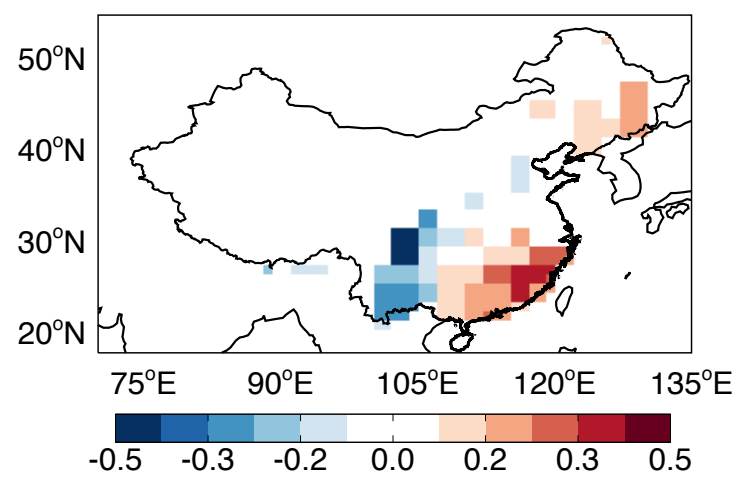

(b) $\triangle$ NPP by TAS changes $(0.07)$

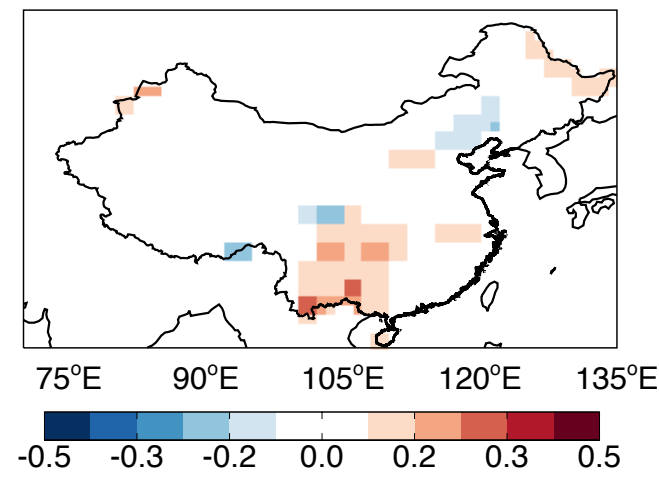

(d) $\triangle$ NPP by SOILM changes $(-0.13)$

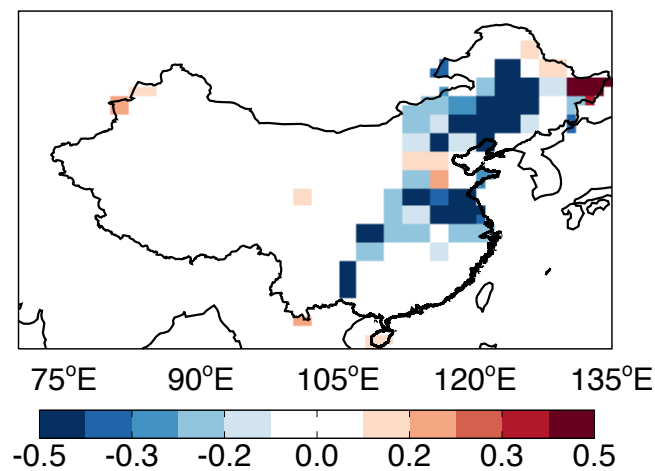

Figure S12. Decomposition of aerosol-induced changes in summer NPP with both aerosol direct and indirect effects. Changes in NPP are caused by aerosol-induced changes in (b) surface air temperature, (c) photosynthetically active radiation (PAR), (d) soil moisture, and (a) the combination of above three effects. Simulations are performed with the offline YIBs vegetation model driven with meteorological forcings simulated with the ModelE2-YIBs climate model (Table S3). The color scale for the first panel is different from the others. The average NPP perturbation over the box domain in (a) is shown in the bracket of each title. Units: $\mathrm{g} \mathrm{C} \mathrm{m}^{-2}$ day $^{-1}$. 


\section{Reference}

Ainsworth, E. A.: Rice production in a changing climate: a meta-analysis of responses to elevated carbon dioxide and elevated ozone concentration, Global Change Biology, 14, 1642-1650, doi:10.1111/J.1365-2486.2008.01594.X, 2008.

An, X. Q., Sun, Z. B., Lin, W. L., Jin, M., and Li, N.: Emission inventory evaluation using observations of regional atmospheric background stations of China, Journal of Environmental Sciences-China, 25, 537-546, doi:10.1016/S1001-0742(12)60082-5, 2013.

Biswas, D. K., Xu, H., Li, Y. G., Sun, J. Z., Wang, X. Z., Han, X. G., and Jiang, G. M.: Genotypic differences in leaf biochemical, physiological and growth responses to ozone in 20 winter wheat cultivars released over the past 60 years, Global Change Biology, 14, 46-59, doi:10.1111/j.1365-2486.2007.01477.x, 2008.

Calatayud, V., Marco, F., Cervero, J., Sanchez-Pena, G., and Sanz, M. J.: Contrasting ozone sensitivity in related evergreen and deciduous shrubs, Environmental Pollution, 158, 3580-3587, doi:10.1016/j.envpol.2010.08.013, 2010.

Feng, Z. Z., Kobayashi, K., and Ainsworth, E. A.: Impact of elevated ozone concentration on growth, physiology, and yield of wheat (Triticum aestivum L.): a meta-analysis, Global Change Biology, 14, 2696-2708, doi:10.1111/J.13652486.2008.01673.X, 2008.

Feng, Z. Z., Niu, J. F., Zhang, W. W., Wang, X. K., Yao, F. F., and Tian, Y.: Effects of ozone exposure on sub-tropical evergreen Cinnamomum camphora seedlings grown in different nitrogen loads, Trees-Structure and Function, 25, 617-625, doi:10.1007/s00468-011-0538-x, 2011a.

Feng, Z. Z., Pang, J., Kobayashi, K., Zhu, J. G., and Ort, D. R.: Differential responses in two varieties of winter wheat to elevated ozone concentration under fully open-air field conditions, Global Change Biology, 17, 580-591, doi:10.1111/j.13652486.2010.02184.x, 2011b.

Foot, J. P., Caporn, S. J. M., Lee, J. A., and Ashenden, T. W.: The effect of long-term ozone fumigation on the growth, physiology and frost sensitivity of Calluna vulgaris, New Phytologist, 133, 503-511, doi:10.1111/J.1469-8137.1996.Tb01918.X, 1996.

Fu, Y., Zhao, T., Sun, J., Cao, Y., Hu, Y., Xu, L., and Shi, Y.: Effects of Elevated Ozone Concentration on Maize Photosynthesis and Grain Quality, Acta Agriculturae Boreali-Sinica, 23, 120-124, doi:10.7668/hbnxb.2008.06.028, 2008.

Grantz, D. A., Vu, H. B., Tew, T. L., and Veremis, J. C.: Sensitivity of Gas Exchange Parameters to Ozone in Diverse C-4 Sugarcane Hybrids, Crop Science, 52, 12701280, doi:10.2135/Cropsci2011.08.0413, 2012.

Han, S. Q., Zhang, M., Zhao, C. S., Lu, X. Q., Ran, L., Han, M., Li, P. Y., and Li, X. J.: Differences in ozone photochemical characteristics between the megacity Tianjin and its rural surroundings, Atmospheric Environment, 79, 209-216, doi:10.1016/j.atmosenv.2013.06.045, 2013.

He, X. Y., Fu, S. L., Chen, W., Zhao, T. H., Xu, S., and Tuba, Z.: Changes in effects of ozone exposure on growth, photosynthesis, and respiration of Ginkgo biloba in Shenyang urban area, Photosynthetica, 45, 555-561, doi:10.1007/s11099-007-0095$0,2007$. 
Li, J., Wang, Z. F., Akimoto, H., Gao, C., Pochanart, P., and Wang, X. Q.: Modeling study of ozone seasonal cycle in lower troposphere over east Asia, J. Geophys. Res., 112, D22s25, doi:10.1029/2006jd008209, 2007.

Lin, W. L., Xu, X. B., Zheng, X. D., Dawa, J., Baima, C., and Ma, J.: Two-year measurements of surface ozone at Dangxiong, a remote highland site in the Tibetan Plateau, Journal of Environmental Sciences-China, 31, 133-145, doi:10.1016/j.jes.2014.10.022, 2015.

Lombardozzi, D., Sparks, J. P., and Bonan, G.: Integrating O3 influences on terrestrial processes: photosynthetic and stomatal response data available for regional and global modeling, Biogeosciences, 10, 6815-6831, doi:10.5194/bg-10-6815-2013, 2013.

Ma, Z. Q., Wang, Y. S., Zhang, X. L., and Xu, J.: Comparison of ozone between Beijing and downstream area, Environmental Science, 32, 924-929, 2011.

Mulchi, C. L., Slaughter, L., Saleem, M., Lee, E. H., Pausch, R., and Rowland, R.: Growth and Physiological-Characteristics of Soybean in Open-Top Chambers in Response to Ozone and Increased Atmospheric Co2, Agriculture Ecosystems \& Environment, 38, 107-118, doi:10.1016/0167-8809(92)90172-8, 1992.

Nali, C., Paoletti, E., Marabottini, R., Della Rocca, G., Lorenzini, G., Paolacci, A. R., Ciaffi, M., and Badiani, M.: Ecophysiological and biochemical, strategies of response to ozone in Mediterranean evergreen broadleaf species, Atmospheric Environment, 38, 2247-2257, doi:10.1016/j.atmosenv.2003.11.043, 2004.

Pang, J., Kobayashi, K., and Zhu, J. G.: Yield and photosynthetic characteristics of flag leaves in Chinese rice (Oryza sativa L.) varieties subjected to free-air release of ozone, Agriculture Ecosystems \& Environment, 132, 203-211, doi:10.1016/j.agee.2009.03.012, 2009.

Sitch, S., Cox, P. M., Collins, W. J., and Huntingford, C.: Indirect radiative forcing of climate change through ozone effects on the land-carbon sink, Nature, 448, 791-794, doi:10.1038/Nature06059, 2007.

Taylor, M. D., Sinn, J. P., Davis, D. D., and Pell, E. J.: The impact of ozone on a salt marsh cordgrass (Spartina alterniflora), Environmental Pollution, 120, 701-705, 2002.

Wang, Q. Y., Gao, R. S., Cao, J. J., Schwarz, J. P., Fahey, D. W., Shen, Z. X., Hu, T. F., Wang, P., Xu, X. B., and Huang, R. J.: Observations of high level of ozone at Qinghai Lake basin in the northeastern Qinghai-Tibetan Plateau, western China, Journal of Atmospheric Chemistry, 72, 19-26, doi:10.1007/s10874-015-9301-9, 2015.

Wang, Y., Zhang, Y., Hao, J., and Luo, M.: Seasonal and spatial variability of surface ozone over China: contributions from background and domestic pollution, Atmospheric Chemistry and Physics, 11, 3511-3525, doi:10.5194/acp-11-3511-2011, 2011.

Wang, Y. H., Hu, B., Tang, G. Q., Ji, D. S., Zhang, H. X., Bai, J. H., Wang, X. K., and Wang, Y. S.: Characteristics of ozone and its precursors in Northern China: A comparative study of three sites, Atmospheric Research, 132, 450-459, doi:10.1016/j.atmosres.2013.04.005, 2013.

Wittig, V. E., Ainsworth, E. A., and Long, S. P.: To what extent do current and projected increases in surface ozone affect photosynthesis and stomatal conductance of trees? 
A meta-analytic review of the last 3 decades of experiments, Plant Cell and Environment, 30, 1150-1162, doi:10.1111/J.1365-3040.2007.01717.X, 2007.

Xu, J., Zhang, X. L., Xu, X. F., Zhao, X. J., Meng, W., and Pu, W. W.: Measurement of surface ozone and its precursors in urban and rural sites in Beijing, Second International Conference on Mining Engineering and Metallurgical Technology (Memt 2011), 2, 255-261, doi:10.1016/j.proeps.2011.09.041, 2011.

$\mathrm{Xu}, \mathrm{S} ., \mathrm{He}, \mathrm{X}$., Chen, W., Huang, Y., Zhao, Y., and Li, B.: Differential sensitivity of four urban tree species to elevated O3, Urban Forestry \& Urban Greening, 14, 1166-1173, doi:10.1016/j.ufug.2015.10.015, 2015.

Xue, L. K., Wang, T., Zhang, J. M., Zhang, X. C., Deliger, Poon, C. N., Ding, A. J., Zhou, X. H., Wu, W. S., Tang, J., Zhang, Q. Z., and Wang, W. X.: Source of surface ozone and reactive nitrogen speciation at Mount Waliguan in western China: New insights from the 2006 summer study, J. Geophys. Res., 116, D07306, doi:10.1029/2010jd014735, 2011.

Yuan, X. Y., Calatayud, V., Jiang, L. J., Manning, W. J., Hayes, F., Tian, Y., and Feng, Z. Z.: Assessing the effects of ambient ozone in China on snap bean genotypes by using ethylenediurea (EDU), Environmental Pollution, 205, 199-208, doi:10.1016/j.envpol.2015.05.043, 2015.

Zhang, L., Su, B. Y., Xu, H., and Li, Y. G.: Growth and photosynthetic responses of four landscape shrub species to elevated ozone, Photosynthetica, 50, 67-76, doi:Doi 10.1007/S11099-012-0004-Z, 2012a.

Zhang, W. W., Feng, Z. Z., Wang, X. K., and Niu, J. F.: Responses of native broadleaved woody species to elevated ozone in subtropical China, Environmental Pollution, 163, 149-157, doi:10.1016/j.envpol.2011.12.035, 2012 b.

Zhang, Y. H., Hu, M., Zhong, L. J., Wiedensohler, A., Liu, S. C., Andreae, M. O., Wang, W., and Fan, S. J.: Regional Integrated Experiments on Air Quality over Pearl River Delta 2004 (PRIDE-PRD2004): Overview, Atmospheric Environment, 42, 61576173, doi:10.1016/j.atmosenv.2008.03.025, 2008.

Zheng, Y., Zhang, J., Wu, R., Zhao, Z., and Hu, C.: Effects of ozone stress on photosynthesis and physiological characteristics of winter wheat in northern China, Journal of Agro-Environment Science, 29, 1429-1436, 2010. 